\title{
A POSITIVE MUSCL-HANCOCK SCHEME FOR IDEAL MAGNETOHYDRODYNAMICS
}

\author{
K. WAAGAN
}

\begin{abstract}
We present a highly robust second order accurate scheme for the Euler equations and the ideal MHD equations. The scheme is of predictor-corrector type, with a MUSCL scheme following as a special case. The crucial ingredients are an entropy stable approximate Riemann solver and a new spatial reconstruction that ensures positivity of mass density and pressure. For multidimensional MHD, a new discrete form of the Powell source terms is vital to ensure the stability properties. The numerical examples show that the scheme has superior stability compared to standard schemes, while maintaining accuracy. In particular, the method can handle very low values of pressure (i.e. low plasma $\beta$ or high Mach numbers) and low mass densities.
\end{abstract}

\section{INTRODUCTION}

This paper is concerned with the nonlinear stability of numerical schemes for the equations of ideal magnetohydrodynamics (MHD). Ideal MHD is a fluid model of a plasma with no resistivity and no net charge. As a model it has been very succesful in describing astrophysical plasmas and space physics. The governing equations are a system of conservation laws for the densities of mass $\rho$, linear momentum $\rho \mathbf{u}$, energy $E$ and magnetic field $\mathbf{B}$. They can be written as (letting $I_{3}$ denote the $3 \times 3$ identity matrix)

$$
\begin{aligned}
& \rho_{t}+\nabla \cdot(\rho \mathbf{u})=0, \\
& (\rho \mathbf{u})_{t}+\nabla \cdot\left(\rho \mathbf{u} \otimes \mathbf{u}+\left(p+\frac{1}{2}|\mathbf{B}|^{2}\right) I_{3}-\mathbf{B} \otimes \mathbf{B}\right)=0, \\
& E_{t}+\nabla \cdot\left[\left(E+p+\frac{1}{2}|\mathbf{B}|^{2}\right) \mathbf{u}-(\mathbf{B} \cdot \mathbf{u}) \mathbf{B}\right]=0, \\
& \mathbf{B}_{t}+\nabla \cdot(\mathbf{B} \otimes \mathbf{u}-\mathbf{u} \otimes \mathbf{B})=0, \\
& \nabla \cdot \mathbf{B}=0,
\end{aligned}
$$

with an internal energy $e$ given by $E=\rho e+\frac{1}{2} \rho \mathbf{u}^{2}+\frac{1}{2} \mathbf{B}^{2}$, and the pressure given by the equation of state $p=p(\rho, e)$. The system fits the generic form of a conservation law $U_{t}+\nabla \cdot \mathbf{F}(U)=0$, except for the restriction on $\nabla \cdot \mathbf{B}$. However, if this restriction is satisfied at the initial time $t=0$, it automatically holds at later times $t>0$ for the exact solution. Since solutions generally have shocks and contact discontininuities, one should look for weak solutions, and augment the system with the entropy inequality

$$
(\rho \phi(s))_{t}+\nabla \cdot(\rho \mathbf{u} \phi(s)) \leq 0
$$

where $\phi$ is any smooth convex and nonincreasing function, and the entropy $s$ is defined by

$$
d e+p d\left(\frac{1}{\rho}\right)=T d s
$$

for some temperature $T(\rho, e)>0$. The entropy inequality means that we apply the second law of thermodynamics to exclude unphysical shock waves.

The ideal MHD system is hyperbolic provided

$$
p^{\prime} \equiv\left(\frac{\partial p}{\partial \rho}\right)_{s}>0
$$

where the subscript $s$ means that the partial derivative is taken with $s$ constant. We also assume that $p(\rho, e)>0$ as long as $\rho>0$ and $e>0$. Hence, a natural stability criterion for a numerical scheme is that $\rho, p$ and $e$ should remain positive numbers, if they are initially. Violations of this 
basically lead to the breakdown of the simulation. It is sufficient to have $\rho>0$ and $\rho e>0$, or equivalently

$$
\rho>0, \quad E-\frac{1}{2} \rho \mathbf{u}^{2}-\frac{1}{2} \mathbf{B}^{2}>0 .
$$

These inequalities define a convex set in the space of conserved variables $U=(\rho, \rho \mathbf{u}, E, \mathbf{B})$. Stronger criteria than positive internal energy are given by imposing discrete versions of the entropy inequality (1.2), or at least a sharp lower bound on $s$. A third important criterion is to avoid spurious oscillations near sharp gradients.

Consider a system of conservation laws in one spatial dimension (we consider the $x$-dimension) $U_{t}+F(U)_{x}=0$. The numerical schemes we will consider are of the finite volume type

$$
\mathcal{S}_{\Delta t} U_{i}=U_{i}-\frac{\Delta t}{h}\left(\mathcal{F}_{i+\frac{1}{2}}-\mathcal{F}_{i-\frac{1}{2}}\right),
$$

where $U_{i}$ are averages over intervals (or 'cells') of length $h$ indexed by $i$ at some time $t$, and the operator $\mathcal{S}_{\Delta t}$ updates the cell averages to time $t+\Delta t$. The numerical fluxes $\mathcal{F}$ are evaluated at the cell interfaces, hence $U$ is conserved and we say the scheme is conservative. First order accurate schemes can be given by

$$
\mathcal{F}_{i+\frac{1}{2}}=\mathcal{F}\left(U_{i}, U_{i+1}\right) .
$$

For MHD several such fluxes are in use, and they are typically given by an approximate Riemann solver (see [7], [26], [19]). We will use the 3-wave approximate Riemann solver of [8]-[9], denoted HLL3R. This numerical flux guarantees a priori that the scheme preserves the positivity of $\rho$ and $e$, and that a discrete version of the entropy inequality holds. Other fluxes with similar properties are given in [18], [23], [17], [20], [21] among others. The mathematical results of this paper are valid for any numerical flux $\mathcal{F}$ ensuring the positivity of (1.6)-(1.7).

These numerical fluxes are building blocks in higher order accurate schemes. The other basic building blocks are typically the spatial reconstruction of states based on the cell averages, and a time integration scheme. The stability properties of the first order schemes are not automatically inherited by the higher order schemes. For one thing, some restriction on the reconstructed states must be imposed to control spurious oscillations. This is the basis of TVD schemes and WENO schemes (see [26], [19]). It is technically complicated to prove a discrete entropy inequality for the higher order schemes $([5],[13])$, and in practice it seems sufficient to have this property for the first order scheme. In any case, TVD and WENO type schemes will revert more or less to the first order scheme near a shock. Finally, even with these considerations made, the positivity property may fail to hold, but, as demonstrated in [24], [3] and [4], positivity can be retained by further restricting the reconstructed states. This idea is carried out here for a second order accurate scheme for ideal MHD. The case of the Euler equations follows by setting $\mathbf{B}=0$.

The extension to more than one space dimension will be performed in a straightforward manner here, by employing uniform Cartesian grids. Stability results from one dimension tend to carry over fairly easily, except that smaller timesteps may be required. For ideal MHD however, there is an obstacle in generalising results from one dimension. The restriction $\nabla \cdot \mathbf{B}=0$ implies that the longitudinal component of $\mathbf{B}, B_{n}$, must be constant for one-dimensional data. When onedimensional schemes are employed in a multidimensional setting, violations of this constraint have to be somehow dealt with. We will use the following approach of [25], consisting of modifying the evolution equation for $\mathbf{B}$ to

$$
\mathbf{B}_{t}+\nabla \cdot(\mathbf{B} \otimes \mathbf{u}-\mathbf{u} \otimes \mathbf{B})-\mathbf{u} \nabla \cdot \mathbf{B}=0 .
$$

This yields a version of the Powell system for MHD. In its original version, also the momentum and energy equations were modified. The HLL3R solver of [8]-[9] has the useful property of being consistent, positive, and to satisfy a discrete entropy inequality also for this more general system. Generalisation of other interesting approximate Riemann solvers to allow Powell terms is possible ([16]). It should be noted that the nonconservative term may lead to errors of a few percent when strong shocks are present, as demonstrated in [27]. An alternative to using (1.8) is the 
staggered mesh (or constrained transport) approach (reviewed in [27]), which in one dimension essentially means evaluating $B_{n}$ at the cell interface instead of as a cell average. It is not clear, as far as we know, whether this leads to provably positive schemes. However, it has the advantage of guaranteeing that $\nabla \cdot \mathbf{B}=0$ to approximation order in smooth regions. We will not attempt to dampen eventual spurious values of $\nabla \cdot \mathbf{B}$ in our test runs, since the role of such methods in numerical stability is unclear. We remark though, that our methods are compatible with the projection method of [10], and the parabolic cleaning method of [22] and [14]. Equation (1.8) implies that

$$
(\nabla \cdot \mathbf{B})_{t}+\nabla \cdot(\mathbf{u} \nabla \cdot \mathbf{B})=0
$$

hence errors in $\nabla \cdot \mathbf{B}$ should be advected with the flow. This may be regarded as a form of cleaning of errors in $\nabla \cdot \mathbf{B}$.

This paper is organised in the following way: Next, we present the underlying MUSCL-Hancock scheme. In Section 3 we derive modifications that will ensure that this scheme is positive in one dimension (i.e. when $B_{n}$ is constant). In Section 4 we extend the positivity result to the multidimensional case (i.e. with variable $B_{n}$ ). Sections 3 and 4 both end with relevant numerical examples. Section 5 contains the main conclusions.

\section{The MUSCL-HANCOCK METHOD}

We now detail the conservative MUSCL-Hancock scheme, introduced in [28] (see also [26]), which generalises a first order finite volume scheme (1.6) to second order accuracy. Later a nonconservative version will be presented. Let $W$ denote the primitive variables $(\rho, \mathbf{u}, \mathbf{B}, p)$. For smooth data, the equations can be rewritten as $W_{t}+A(W) W_{x}=0$ for a matrix $A(W)$. The MUSCL-Hancock schemes goes as follows.

(1) Evaluate discrete differences $d W_{i}$. For oscillation control, we use the MC-limiter (monotonised central limiter), so for each component of $W_{i}$ we take

$$
d W_{i}=\sigma_{i} \min \left(2\left|W_{i+1}-W_{i}\right|, \frac{1}{2}\left|W_{i+1}-W_{i-1}\right|, 2\left|W_{i}-W_{i-1}\right|\right)
$$

with

$$
\sigma_{i}=\left\{\begin{array}{l}
1, \quad W_{i+1}-W_{i}>0, W_{i}-W_{i-1}>0 \\
-1, \quad W_{i+1}-W_{i}<0, W_{i}-W_{i-1}<0 \\
0, \quad \text { otherwise. }
\end{array}\right.
$$

(2) Limit $d W_{i}$ to ensure positivity as outlined below. This gives new differences $D W_{i}$.

(3) Prediction step: Evaluate

$$
W_{i}^{c}=W_{i}-\frac{\Delta t}{2 h} A\left(W_{i}\right) D W_{i}
$$

(4) Evaluate the cell edge values

$$
W_{i}^{-}=W_{i}^{c}-\frac{1}{2} D W_{i}, \quad W_{i}^{+}=W_{i}^{c}+\frac{1}{2} D W_{i} .
$$

(5) Use the cell edge values as input to the numerical flux in the conservative scheme.

$$
\mathcal{S}_{\Delta t} U_{i}=U_{i}-\frac{\Delta t}{h}\left(\mathcal{F}\left(U_{i}^{+}, U_{i+1}^{-}\right)-\mathcal{F}\left(U_{i-1}^{+}, U_{i}^{-}\right)\right) .
$$

The positivity result we derive next, does not depend on our specific choice (2.1) of gradient limiter. The spatial reconstruction step (2.4) may be replaced with a conservative reconstruction (i.e. such that $\left.\frac{1}{2}\left(U^{-}+U^{+}\right)=U^{c}\right)$ as follows

$$
\begin{array}{r}
\rho^{ \pm}=\rho^{c} \pm \frac{1}{2} D \rho, \quad \mathbf{u}^{ \pm}=\mathbf{u}^{c} \pm \frac{\rho^{\mp}}{2 \rho^{c}} D \mathbf{u}, \quad \mathbf{B}^{ \pm}=\mathbf{B}^{c} \pm \frac{1}{2} D \mathbf{B} \\
p^{ \pm}=p^{c} \pm \frac{1}{2} D p-(\gamma-1)\left(\frac{\rho^{+} \rho^{-}}{8 \rho} D \mathbf{u}^{2}-\frac{1}{8} D \mathbf{B}^{2}\right),
\end{array}
$$


assuming the ideal gas law $p=(\gamma-1) \rho e$. We will refer to this as the $U$-reconstruction, and (2.4) as the $W$-reconstruction. A convenient third option is what we will call the $p$-reconstruction, where we take a conservative slope for the momentum, but set

$$
p^{ \pm}=p^{c} \pm \frac{1}{2} D p
$$

In [4] the positivity of a MUSCL-Hancock scheme is analysed with the Euler equations as an example. The prediction step (2.3) was in [4] replaced with

$$
U_{i}^{c}=U_{i}-\frac{\Delta t}{2 h}\left(F\left(U_{i}^{+}\right)-F\left(U_{i}^{-}\right)\right) .
$$

Using primitive variables as the basis for reconstructing the states was recommended for example in [12]. It ensures that material contact discontinuities are reproduced exactly. Alternatively, one can use that the primitive form $W_{t}+A(W) W_{x}=0$ may be diagonalised as

$$
\left(R_{i}^{j}\right)_{t}+\lambda^{j}\left(R_{i}^{j}\right)_{x}=0, \quad j=1,2, \ldots, d
$$

with $W_{i}=X_{i} R_{i}$. The matrix $X_{i}$ is given by the eigenvectors $X_{i}^{j}$ of $A\left(W_{i}\right)$. The reconstructed gradients may then be evaluated using the relation $D W_{i}=X_{i} D R_{i}$, given gradients $D R_{i}$, and we get

$$
W_{i}^{ \pm}=X_{i} R_{i}^{ \pm}=X_{i}\left(R_{i}-\frac{\Delta t}{2 h} \lambda R_{i} \pm \frac{1}{2} D R_{i}\right)=W_{i}-\frac{\Delta t}{2 h} A\left(W_{i}\right) D W_{i} \pm \frac{1}{2} D W_{i},
$$

where $\lambda$ is the diagonal matrix having the eigenvalues $\lambda^{j}$ as entries. The estimates we need to ensure positivity are most conveniently stated for $D W$, but they are also valid for the characteristic reconstruction (2.11). For ideal MHD the characteristic decomposition is costly and complicated due to the nonstrict hyperbolicity, so we will evaluate $d W$ directly from (2.1) in our examples.

\section{Positivity analysis in One Spatial Dimension}

We first derive a positive version of the conservative MUSCL-Hancock scheme (2.5). For convenience we restrict ourselves to the case that $\gamma-1=p /(\rho e)$ can be considered fixed, but this restriction is easily relaxed. Our starting point is the following proposition, essentially due to [3]. We will say that a scheme $S_{\Delta t}$ preserves an invariant region $\Omega$ if

$$
U_{i} \in \Omega \quad \forall i \quad \Rightarrow \quad S_{\Delta t} U_{i} \in \Omega \quad \forall i .
$$

This typically only holds under a CFL condition $\Delta t<\tau(U) h$ where $\tau$ is a function of the sequence of cell averages $\left\{U_{i}\right\}_{i}$.

Proposition 3.1. The MUSCL-Hancock scheme (2.5) preserves the convex invariant region $\Omega$ if i) The scheme (1.6)-(1.7) preserves $\Omega$ for all $\Delta t<\tau\left(U_{i}\right) h$, and

ii) For some $\alpha \in\left(0, \frac{1}{3}\right]$

$$
U_{i}^{+} \in \Omega, \quad U_{i}^{-} \in \Omega \text { and } \quad U_{i}^{*}=\frac{1}{1-2 \alpha}\left(U-\alpha\left(U_{i}^{+}+U_{i}^{-}\right)\right) \in \Omega,
$$

and $\Delta t<\alpha \tau(\tilde{U}) h$, where $\tilde{U}$ is the sequence

$$
\left\{\ldots, U_{i-1}^{-}, U_{i-1}^{*}, U_{i-1}^{+}, U_{i}^{-}, U_{i}^{*}, U_{i}^{+}, U_{i+1}^{-}, U_{i+1}^{*}, U_{i+1}^{+}, \ldots\right\} .
$$

The optimal CFL condition is given by $\alpha=\frac{1}{3}$. This means we need to take about three times smaller time steps compared to the first order scheme. Also, the CFL condition is implicitly defined. In practice we find that our schemes work well at much higher CFL numbers than theoretically accounted for. The practical significance of the result is to provide bounds on the reconstructed values $U^{ \pm}$rather than on $\Delta t$. 
Proof. The method can be written as a convex combination of first order schemes. Let $F^{-}=$ $\mathcal{F}\left(U_{i-1}^{+}, U_{i}^{-}\right)$and $F^{+}=\mathcal{F}\left(U_{i}^{+}, U_{i+1}^{-}\right)$, which allows us to drop the index $i$ for readability. Using the definition of $U^{*}$, we get

$$
\begin{aligned}
\mathcal{S}_{\Delta t} U=U-\frac{\Delta t}{h}\left(F^{+}-F^{-}\right) & =\alpha U^{-}-\frac{\Delta t}{h}\left(\mathcal{F}\left(U^{-}, U^{+}\right)-F^{-}\right) \\
+(1-2 \alpha) U^{*} & -\frac{\Delta t}{h}\left(\mathcal{F}\left(U^{*}, U^{+}\right)-\mathcal{F}\left(U^{-}, U^{+}\right)\right) \\
& +\alpha U^{+}-\frac{\Delta t}{h}\left(F^{+}-\mathcal{F}\left(U^{*}, U^{+}\right)\right) .
\end{aligned}
$$

We rewrite this as

$$
\begin{aligned}
\mathcal{S}_{\Delta t} U & =\alpha\left(U^{-}-\frac{\Delta t}{h \alpha}\left(\mathcal{F}\left(U^{-}, U^{+}\right)-F^{-}\right)\right) \\
& +(1-2 \alpha)\left(U^{*}-\frac{\Delta t}{h(1-2 \alpha)}\left(\mathcal{F}\left(U^{*}, U^{+}\right)-\mathcal{F}\left(U^{-}, U^{+}\right)\right)\right) \\
& +\alpha\left(U^{+}-\frac{\Delta t}{h \alpha}\left(F^{+}-\mathcal{F}\left(U^{*}, U^{+}\right)\right)\right) .
\end{aligned}
$$

Each line is a first order update of a cell average with time step sizes $\Delta t / \alpha$ and $\Delta t /(1-2 \alpha)$. This means that, provided $\Delta t$ is chosen as prescribed, $\mathcal{S}_{\Delta t} U$ is a convex combination of states in $\Omega$. The result then follows by the convexity of $\Omega$.

Remark: This Proposition is only valid for conservative schemes. In the case of multidimensional MHD, the Powell terms result in a nonconservative flux in place of $\mathcal{F}$. We will generalise to the Powell system in the next section.

3.1. Implementation: Positive mass density. We now demonstrate how Proposition 3.1 can be used. The index $i$ is dropped throughout this section for readability. Hence $\rho, U$ etc. is to be understood as generic cell averages $\rho_{i}, U_{i}$ etc. We choose the optimal $\alpha=1 / 3$. First we consider positivity of mass density. The prediction step for $\rho$ is

$$
\rho^{c}=\rho-\frac{\Delta t}{2 h}(u D \rho+\rho D u)
$$

According to Proposition 3.1, it is enough to have:

$$
\min \left(\rho^{-}, \rho^{+}\right)=\rho-\frac{\Delta t}{2 h}(u D \rho+\rho D u)-\frac{1}{2}|D \rho|>0,
$$

and

$$
\rho^{*}=\rho+2 \frac{\Delta t}{2 h}(u D \rho+\rho D u)>0
$$

This yields the sufficient condition that

$$
\rho-\frac{\Delta t}{h}(|u||D \rho|+\rho|D u|)-\frac{1}{2}|D \rho|>0 .
$$

Set $|D \rho|<l \rho$ and $|D u|<l \frac{h}{\Delta t}$, for some $l$ to be determined. Assuming the reasonable CFL condition $|u| \leq \frac{h}{\Delta t}$, we end up with $l=\frac{1}{2}$, so $|D \rho|<\frac{\rho}{2}$ and $|D u|<\frac{h}{2 \Delta t}$.

3.2. Implementation: Positive pressure. Ensuring that pressure also stays positive is more involved. Define $\Delta W=\frac{\Delta t}{2 h} A(W) D W$, so that

$$
W^{c}=W-\Delta W
$$


Hence $\Delta W$ is given as

$$
\begin{aligned}
\Delta \rho & =\frac{\Delta t}{2 h}(u D \rho+\rho D u) \\
\Delta u & =\frac{\Delta t}{2 h}\left(u D u+\frac{D p+B_{\perp} \cdot D B_{\perp}}{\rho}\right) \\
\Delta u_{\perp} & =\frac{\Delta t}{2 h}\left(u D u_{\perp}-\frac{B_{n} D B_{\perp}}{\rho}\right) \\
\Delta B_{n} & =0 \\
\Delta B_{\perp} & =\frac{\Delta t}{2 h}\left(u D B_{\perp}+D u B_{\perp}-B_{n} D u_{\perp}\right) \\
\Delta p & =\frac{\Delta t}{2 h}(u D p+\gamma p D u) .
\end{aligned}
$$

To separate between longitudinal and transversal components, we introduced here the notation $\mathbf{u}=\left(u, u_{\perp}\right)$ and $\mathbf{B}=\left(B_{n}, B_{\perp}\right)$. Hence $u_{\perp}$ and $B_{\perp}$ are vectors in the $y-z$ plane. Since we are in one dimension we assumed $B_{n}$ to be constant. Proposition 3.1 motivates the following estimates:

Proposition 3.2. The $W$-reconstruction satisfies the conditions of Proposition 3.1 with $\alpha=\frac{1}{3}$ if

$$
|D \rho|<\frac{1}{2} \rho, \quad|D u|<\frac{\Delta x}{(1+\gamma) \Delta t}, \quad|D p|<\frac{p}{1+\gamma}
$$

and

$$
\begin{aligned}
3 \frac{\rho^{c} \rho}{\rho^{*}}(\Delta \mathbf{u})^{2}+3(\Delta \mathbf{B})^{2}+\frac{1}{4}\left(\left(\rho^{c}+\frac{D \rho^{2}}{2 \rho^{*}}\right) D \mathbf{u}^{2}+D \mathbf{B}^{2}\right) \\
\quad+\frac{1}{2} D \rho \mathbf{u} \cdot \Delta \mathbf{u}-\left(\frac{\rho^{c}}{\rho^{*}}+1\right) D \rho D \mathbf{u} \cdot \Delta \mathbf{u}<\frac{p+2 \Delta p}{\gamma-1} .
\end{aligned}
$$

The p-reconstruction satisfies the conditions of Proposition 3.1 with $\alpha=\frac{1}{3}$ if (3.14) holds and

$$
3 \frac{\rho^{c} \rho}{\rho^{*}}(\Delta \mathbf{u})^{2}+3(\Delta \mathbf{B})^{2}+\frac{1}{4}\left(\rho^{c} D \mathbf{u}^{2}+D \mathbf{B}^{2}\right)<\frac{p+2 \Delta p}{\gamma-1} .
$$

For the U-reconstruction, the conditions of Proposition 3.1 with $\alpha=\frac{1}{3}$ are satisfied if

$$
|D \rho|<\frac{1}{2} \rho, \quad|D u|<\frac{\Delta x}{2 \Delta t}, \quad \frac{1}{8}\left(\rho^{c} D \mathbf{u}^{2}+D \mathbf{B}^{2}\right)<\frac{p^{c}-\frac{1}{2}|D p|}{\gamma-1}
$$

and

$$
3 \frac{\rho^{c} \rho}{\rho^{*}}(\Delta \mathbf{u})^{2}+3(\Delta \mathbf{B})^{2}<\frac{p+2 \Delta p}{\gamma-1}
$$

Proof. Consider the W-reconstruction. The conditions (3.14) imply that $\rho^{ \pm}>0, p^{ \pm}>0$ and $\rho^{*}>0$ by arguments like those given in Section 3.1. Note that they also ensure that $p+2 \Delta p>0$, and that $\rho^{c}>0$.

It remains to check that $(\rho e)^{*}>0$, or in terms of conserved quantities, that

$$
3 E-\left(E^{+}+E^{-}\right)-\frac{1}{2} \frac{\left((\rho \mathbf{u})^{*}\right)^{2}}{\rho^{*}}-\frac{1}{2}\left(\mathbf{B}^{*}\right)^{2}>0 .
$$

Note that

$$
(\rho \mathbf{u})^{*}=3 \rho \mathbf{u}-\left(2 \rho^{c} \mathbf{u}^{c}+\frac{1}{2} D \rho D \mathbf{u}\right)=\rho^{*} \mathbf{u}+2 \rho^{c} \Delta \mathbf{u}-\frac{1}{2} D \rho D \mathbf{u} .
$$


Hence we get

$$
\begin{aligned}
\frac{1}{2} \frac{\left((\rho \mathbf{u})^{*}\right)^{2}}{\rho^{*}}= & \frac{1}{2} \rho^{*} \mathbf{u}^{2}+2 \frac{\left(\rho^{c}\right)^{2}}{\rho^{*}}(\Delta \mathbf{u})^{2}+2 \rho^{c} \mathbf{u} \cdot \Delta \mathbf{u} \\
& +\frac{D \rho^{2}}{8 \rho^{*}} D \mathbf{u}^{2}-\frac{1}{2} D \rho \mathbf{u} \cdot D \mathbf{u}-\frac{\rho^{c} D \rho}{\rho^{*}} \Delta \mathbf{u} D \mathbf{u}
\end{aligned}
$$

For the calculation of $E^{+}+E^{-}$, we need

$$
\begin{aligned}
\frac{1}{2} \rho^{+}\left(\mathbf{u}^{+}\right)^{2}+\frac{1}{2} \rho^{-}\left(\mathbf{u}^{-}\right)^{2} & =\rho^{c}\left(\left(\mathbf{u}^{c}\right)^{2}+\frac{1}{4}(D \mathbf{u})^{2}\right)+D \rho \mathbf{u}^{c} \cdot D \mathbf{u} \\
& =\rho^{c}\left(\mathbf{u}^{2}+(\Delta \mathbf{u})^{2}-2 \mathbf{u} \cdot \Delta \mathbf{u}+\frac{1}{4}(D \mathbf{u})^{2}\right)+D \rho(\mathbf{u}-\Delta \mathbf{u}) \cdot D \mathbf{u}
\end{aligned}
$$

Also,

$$
\frac{1}{2}\left(\mathbf{B}^{*}\right)^{2}=\frac{1}{2} \mathbf{B}^{2}+2(\Delta \mathbf{B})^{2}+2 \mathbf{B} \cdot \Delta \mathbf{B}
$$

and

$$
\frac{1}{2}\left(\mathbf{B}^{+}\right)^{2}+\frac{1}{2}\left(\mathbf{B}^{-}\right)^{2}=\left(\mathbf{B}^{c}\right)^{2}+\frac{1}{4}(D \mathbf{B})^{2}=\mathbf{B}^{2}+\Delta \mathbf{B}^{2}-2 \mathbf{B} \cdot \Delta \mathbf{B}+\frac{1}{4} D \mathbf{B}^{2} .
$$

Plugging this into $(3.19)$, the positivity of $(\rho e)^{*}$ follows. We used the relation

$$
\rho^{c}+2 \frac{\left(\rho^{c}\right)^{2}}{\rho^{*}}=3 \frac{\rho^{c} \rho}{\rho^{*}} .
$$

The results for the $p$ - and $U$ - reconstructions follow similarily. For these two cases we have

$$
\begin{aligned}
(\rho \mathbf{u})^{*} & =3 \rho \mathbf{u}-2 \rho^{c} \mathbf{u}^{c}=\rho^{*} \mathbf{u}+2 \rho^{c} \Delta \mathbf{u}, \\
\frac{1}{2} \frac{\left((\rho \mathbf{u})^{*}\right)^{2}}{\rho^{*}} & =\frac{1}{2} \rho^{*} \mathbf{u}^{2}+2 \frac{\left(\rho^{c}\right)^{2}}{\rho^{*}}(\Delta \mathbf{u})^{2}+2 \rho^{c} \mathbf{u} \cdot \Delta \mathbf{u}, \\
\frac{1}{2} \rho^{+}\left(\mathbf{u}^{+}\right)^{2}+\frac{1}{2} \rho^{-}\left(\mathbf{u}^{-}\right)^{2} & =\rho^{c}\left(\mathbf{u}^{c}\right)^{2}+\frac{\rho^{-} \rho^{+}}{4 \rho^{c}}(D \mathbf{u})^{2} .
\end{aligned}
$$

Remark 1: Taking $\alpha=\frac{1}{4}$ replaces (3.15) with the slightly simpler

$$
\frac{\rho^{c} \rho}{\rho^{*}}(\Delta \mathbf{u})^{2}+(\Delta \mathbf{B})^{2}+\frac{1}{8}\left(\left(\rho^{c}+\frac{D \rho^{2}}{4 \rho^{*}}\right) D \mathbf{u}^{2}+D \mathbf{B}^{2}\right)+\frac{D \rho \Delta \rho}{\rho^{*}} D \mathbf{u} \Delta \mathbf{u}<\frac{p+\Delta p}{\gamma-1}
$$

Remark 2: The proof does not rely on any explicit formula for $\Delta W$. By setting $\Delta W=0$, a positive semi-discrete MUSCL scheme follows as a special case. An appropriate Runge-Kutta type time discretisation, such as Heun's method, will yield a positive scheme that is second order in time and space.

Taking $D W=0$, which means that we are back to the first order scheme, satisfies the condition. Noting that, the next step is to find a sharper choice of $D W$. Let us first consider the $W$ reconstruction. The algorithm's outline is as follows: i)Ensure that (3.14) holds, then ii)Ensure that (3.15) holds. Point ii) requires some further estimation.

Define

$$
\begin{aligned}
L(d W)=3 \frac{\tilde{\rho}^{c} \rho}{\tilde{\rho}^{*}}(\Delta \mathbf{u})^{2} & +3(\Delta \mathbf{B})^{2}+\frac{1}{4}\left(\left(\tilde{\rho}^{c}+\frac{D \rho^{2}}{2 \tilde{\rho}^{*}}\right) D \mathbf{u}^{2}+D \mathbf{B}^{2}\right) \\
+ & \frac{1}{2}(D \rho \mathbf{u} \cdot \Delta \mathbf{u})_{+}-\left(\frac{\tilde{\rho}^{c}}{\tilde{\rho}^{*}}+1\right)(D \rho D \mathbf{u} \cdot \Delta \mathbf{u})_{-}
\end{aligned}
$$

with

$$
\tilde{\rho}^{c}=\rho-\frac{\Delta t}{2 h}\left(\rho(D u)_{-}+(u D \rho)_{-}\right) \geq \rho^{c}, \quad \tilde{\rho}^{*}=3 \rho-2 \tilde{\rho}^{c} \leq \rho^{*}
$$




\begin{tabular}{l|c|c|c|c} 
& $W$-rec. & $p$-rec. & $U$-rec. & First order \\
\hline Basic scheme & MHW & MHp & MHU & \\
Positive cell edge values & $\mathrm{MHW}^{ \pm}$ & $\mathrm{MHp}^{ \pm}$ & $\mathrm{MHU}^{ \pm}$ & \\
Positive scheme & $\mathrm{MHWP}$ & $\mathrm{MHpP}$ & MHUP & G1
\end{tabular}

TABLE 1. Summary of the schemes considered in one-dimensional tests.

Also, define

$$
\underline{\rho e}(d W)=\frac{p+2 \frac{\Delta t}{2 h}\left((u d p)_{-}+\gamma p(d u)_{-}\right)}{\gamma-1} .
$$

Then it is sufficient for $(3.15)$ to have $L(D W)<\underline{\rho e}(D W)$. Using this, we end up with the following algorithm for the reconstruction:

(1) Compute $d W$ with a standard limiting procedure such as (2.1).

(2) Limit $d W$ to ensure (3.14)

(3) Compute $L(d W), \rho e(d W)$, and

$$
f(d W)=\sqrt{\frac{\rho e(d W)}{\max (L(d W), \underline{\rho e}(d W))}}
$$

(4) Set $D W=f(d W) d W$, and $\Delta W=f(d W) A(W) d W$

(5) Compute $W^{c}$ and $W^{ \pm}$.

The validity of this algorithm is most easily seen as follows: The right hand side $\rho e$ is nonincreasing in the absolute value of each component of $d W$. For the left hand side we have $L \overline{(\xi} d W) \leq \xi^{2} L(d W)$ for all $\xi \in[0,1]$. This implies

$$
L(D W) \leq f(d W)^{2} L(d W) \leq \underline{\rho e}(d W) \leq \underline{\rho e^{p}}(D W) .
$$

For the $U$ - and $p$-reconstructions the algorithm is essentially the same.

For comparison purposes we also set up a scheme such that $U^{ \pm}$are positive, but with no guarantees for $U^{*}$. This scheme is not provably positive, but is based on a natural and common design principle. For the $W$ - and $p$-reconstructions it is sufficient to have

$$
|D \rho|<\frac{2}{3} \rho, \quad|D u|<\frac{2 \Delta x}{(2+\gamma) \Delta t}, \quad|D p|<\frac{2 p}{2+\gamma} .
$$

For the $U$-reconstruction, we also need

$$
\frac{1}{8}\left(\rho^{c} D \mathbf{u}^{2}+D \mathbf{B}^{2}\right)<\frac{p^{c}-\frac{1}{2}|D p|}{\gamma-1} .
$$

3.3. Numerical tests in one spatial dimension. We now have schemes ready to be tested on hydrodynamics and one-dimensional MHD. Let us denote the basic MUSCL-Hancock schemes with $W, p$ and $U$ reconstructions MHW, MHp and MHU respectively. The positive versions will be denoted MHWP, MHpP and MHUP. We also consider a first order scheme G1 (with $D W=0$ ), and schemes which guarantee the positivity of the cell edge values $U^{ \pm}$, but not positivity in general. The different schemes are summarised in Table 1. The tests focus on specific issues of the new positive schemes, and we refer to [26] for more basic tests of the MUSCL-Hancock scheme. First, we fix some parameters. The time step is chosen according to the following formula

$$
\Delta t=C_{c f l} \frac{h}{\max \left(\sup _{i}\left(\mathbf{u}_{i}+\frac{\left|\mathbf{B}_{i}\right|}{\sqrt{\rho_{i}}}\right), \Lambda\right)},
$$

where $\Lambda$ denotes the maximum over all cell interfaces of the Riemann solver signal velocities of the previous time step (For the first time step, the previous time step is defined as one with $\Delta t=0$ ). We set $C_{c f l}=0.9$, which is of more practical interest than the smaller theoretical value. For each of the limiting inequalities (3.14)-(3.18), we multiply the right hand sides with 0.9 to avoid marginality. The gas is assumed to be ideal in all examples, so the equation of state is $(\gamma-1) \rho e=p$ for a fixed $\gamma \in(1,2]$. 
A POSITIVE MUSCL-HANCOCK SCHEME FOR IDEAL MAGNETOHYDRODYNAMICS

\begin{tabular}{l|cc|cc|cc} 
& MHW & MHWP & MHp & MHpP & MHU & MHUP \\
\hline$A_{\text {crit }}$ & 15.5 & & 10.1 & & 3.6 & \\
$100 v_{\text {err }}$ & 3.07 & 4.32 & 3.02 & 3.31 & 2.89 & 2.93 \\
\hline & MHW $^{ \pm}$ & MHWP & MHp $^{ \pm}$ & MHpP & MHU $^{ \pm}$ & MHUP \\
\hline$A_{\text {crit }}$ & 23.4 & & 11.2 & & $50^{*}$ & \\
$100 v_{\text {err }}$ & 4.80 & 6.14 & 3.14 & 3.49 & 11.1 & 11.8
\end{tabular}

TABLE 2. The nonpositive schemes were tested at the values of $A=A_{\text {crit }}$ that they were marginally stable, and the error in $v$ compared to their positive counterparts at the same $A_{\text {crit }}$. ${ }^{*}$ For $\mathrm{MHU}^{ \pm}$we could not find an $A_{\text {crit }}$, so $A=50$ was chosen as a convenient value.

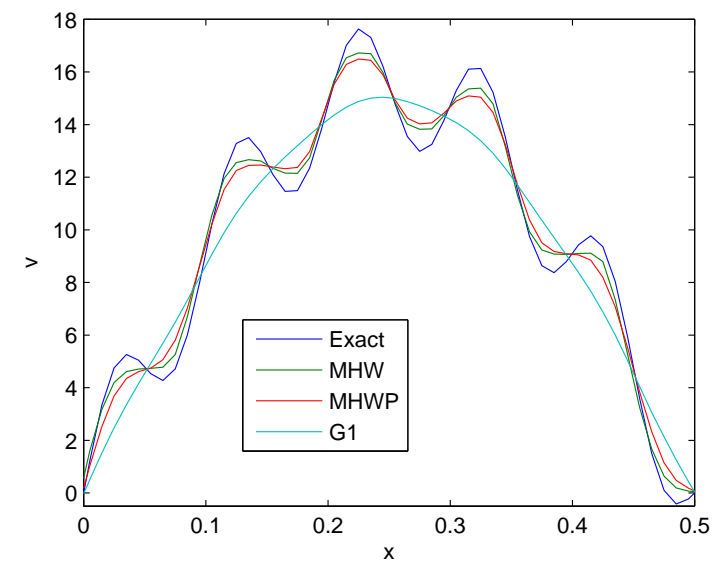

Figure 3.1. Shear test for MHW with $A=15.5$ at $t=0.02$. The results are similar for $0.5<x<1$. At higher $A$, MHW failed.

3.3.1. High Mach shear flow. Our first test, consisting of an advected shear flow, was set up to investigate the accuracy of the positive schemes compared to the basic schemes. Take $\rho=1$, $p=1 / \gamma, \mathbf{B}=0, \gamma=5 / 3$ and

$$
\mathbf{u}=(50, A(\sin 2 \pi x+0.15 \sin 20 \pi x), 0), \quad A>0 .
$$

The strategy was to increase the free parameter $A$ until the nonpositive schemes are marginally stable. We first increased $A$ until MHW was not able to advect the flow for one period (time $t=0.02)$. The resolution was fixed to $h=0.01$, and boundary conditions were periodic. MHW produced a solution at $A=15.5$, while it terminated due to negative pressure values at $A=15.6$ and larger values we tested. Figure 3.1 compares MHW and MHWP at $A=15.5$. The G1 result is also plotted to show how especially the smaller scale is more smeared out. The error with respect to the exact solution in shear velocity $v_{\text {exact }}$ is conveniently measured as the normalised $L^{1}$-error $v_{\text {err }}=\Sigma_{i}\left|v_{i}-v\left(0, x_{i}\right)\right| h / A$. For MHWP the error was 0.0432 , while for MHW it was 0.0307 . It is not surprising that MHWP gave a larger error in this critical case, but it was still much less than the error of G1, which was 0.0913 .

We performed the same test with the scheme given by (3.35), denoted $\mathrm{MHW}^{ \pm}$. This scheme produced negative pressures at $A=23.5$ and higher, hence having positive cell edge states only increased the marginal $A$ with about $50 \%$. At $A=23.4 \mathrm{MHWP}$ produced $v_{\text {err }}=0.0614$, while $\mathrm{MHW}^{ \pm}$produced $v_{\text {err }}=0.0480$

The same type of testing was performed on the other schemes. First, a critical amplitude $A=A_{\text {crit }}$ was found, and then the positive scheme was compared to its nonpositive counterpart. the results are summarised in Table 2. We observe the same tendencies with all schemes, except that $\mathrm{MHU}^{ \pm}$, given by (3.35)-(3.36), appeared very stable on this case. The scheme $\mathrm{MHp}^{ \pm}$, given by (3.35), had only about $10 \%$ better stability range than MHp. 


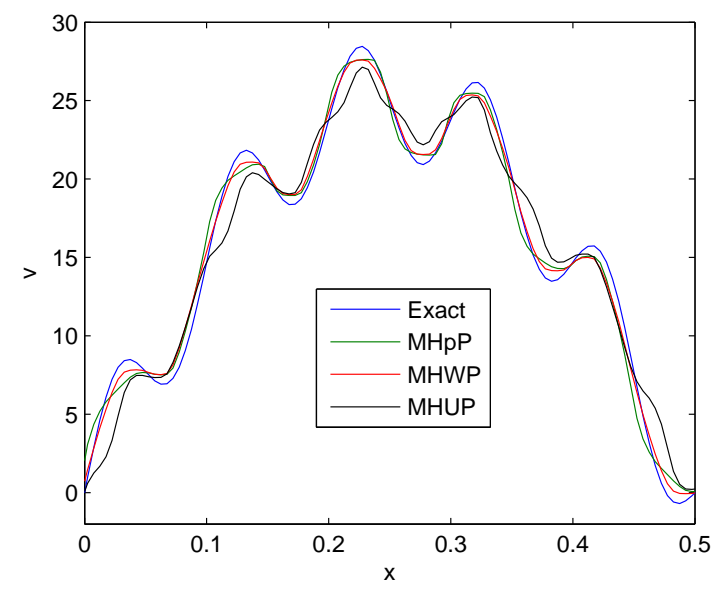

FiguRE 3.2. Shear test with $A=25$ at $t=0.02$ with $h=0.005$. The results are similar for $0.5<x<1$. All the basic schemes MHU, MHp and MHW failed at this $A$-value.

\begin{tabular}{l|ccc}
$h$ & MHWP, A=1 & MHW A=15.5 & MHWP, A=15.5 \\
\hline 0.02 & 7.32 & 7.82 & 8.51 \\
0.01 & 2.44 & 3.07 & 4.32 \\
0.05 & 0.64 & 1.09 & 1.27 \\
0.025 & 0.19 & 0.39 & 0.48
\end{tabular}

TABLE 3. Shear test: Errors given as $100 v_{\text {err }}$ at $t=0.02$.

\begin{tabular}{l|ccc}
$h$ & MHUP & MHpP & MHWP \\
\hline 0.02 & 10.2 & 9.30 & 8.91 \\
0.01 & 9.79 & 6.31 & 5.82 \\
0.05 & 5.27 & 2.99 & 2.33 \\
0.025 & 1.84 & 1.18 & 0.98
\end{tabular}

TABLE 4. Shear test: Errors given as $100 v_{\text {err }}$ at $A=25$ for different schemes at $t=0.02$. All the basic schemes MHU, MHp and MHW failed at this $A$.

Tables 3-4 show errors from refinements studies with varying $A$. At $A=1$ we noted little difference between the schemes, so only the results from MHWP are shown. At $A=15.5 \mathrm{MHW}$ and MHWP still converge at about the same rate with the errors of MHWP slightly larger. Convergence was slower than at $A=1$. The next step is to compare the positive schemes MH.P. Table 4 compares the errors of these schemes at $A=25$, revealing that MHUP is somewhat less accurate. In Figure 3.2, showing the output of different schemes for a fixed resolution, MHWP may be seen to best reproduce the shape of the shear wave, especially compared to MHUP. Since MHUP is also fairly complicated to calculate, we focus on MHWP and MHpP from here onwards.

The main conclusions from this test are that the limiting prescribed by Proposition 3.2 significantly improves the stability of the basic MUSCL-Hancock schemes, while maintaining the accuracy of the original schemes. We also demonstrated that simply having the cell edges $U^{ \pm}$ positive is an insufficient stability criterion, at least for the $W$ - and $p$-reconstructions.

3.3.2. Brio-Wu shock tube. It is also important to demonstrate that the new schemes can resolve shocks and contact discontinuities well. We consider shock tube initial data from [11] that has become a standard test case. The initial data are given by $U=U_{l}$ for $x<0.5$, and $U=U_{r}$ for 

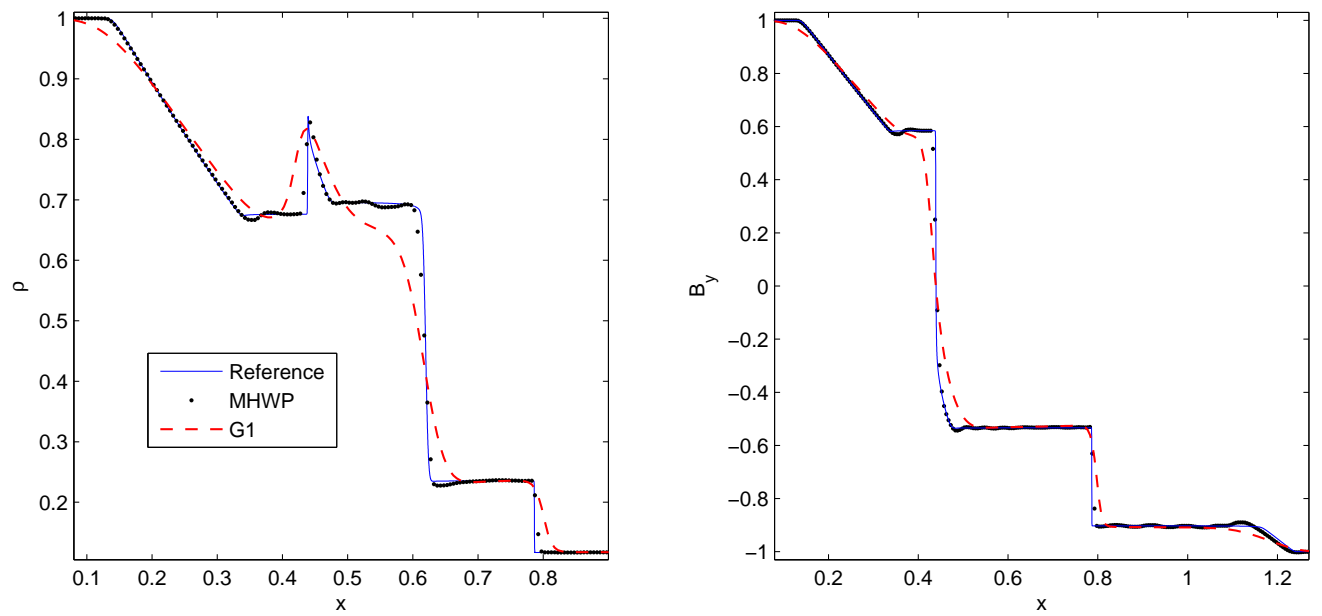

Figure 3.3. Brio-Wu shock tube test as computed by G1 and MHWP. The resolution was $h=0.005$, and $\rho$ and $B_{y}$ are plotted at $t=0.2$. The reference solution results from a computation with $h=10^{-4}$ using the first order scheme G1.

$x>0.5$, with $\gamma=2$ and

$$
\begin{array}{r}
\rho_{l}=1, \mathbf{u}_{l}=0, \mathbf{B}_{l}=(0.75,1,0), p_{l}=1 \\
\rho_{r}=0.125, \mathbf{u}_{r}=0, \mathbf{B}_{r}=(0.75,-1,0), p_{r}=0.1 .
\end{array}
$$

All the second order schemes produced very similar results on this case. Since the TVD-type reconstruction used as basis for the second order schemes already limits the gradients strongly near discontinuities, it is not surprising that the extra limiting applied in the positive schemes has little influence in this problem. Data from MHWP and G1 are shown in Figure 3.3. Note the strong improvement to the first order scheme G1.

3.3.3. Vacuum shock tube test. This test is from [9] (see also [6] and [7] for hydrodynamics), where the ability of the flux HLL3R to handle vacuum data was demonstrated. We show here that our approach extends this ability to higher order schemes. The initial data are given by $U=U_{l}$ for $x<0.5$, and $U=U_{r}$ for $x>0.5$, with $\gamma=2$ and

$$
\begin{array}{r}
\rho_{l}=0, \mathbf{u}_{l}=0, \mathbf{B}_{l}=0, p_{l}=0 \\
\rho_{r}=1, \mathbf{u}_{r}=0, \mathbf{B}_{r}=(0,1,0), p_{r}=0.5 .
\end{array}
$$

Positivity is critical for this problem. We specify that in vacuum cells $D W$ was set to zero. Figure 3.4 shows results by MHWP and G1. A similar undershoot at the rarefaction tail was also reported in [7] for a MUSCL scheme. The density profile from $\mathrm{MHpP}$ is not plotted as it is indiscernible from that of MHWP. The base scheme MHp failed here due to a negative pressure value. The MHW run stagnated due to a very high sound speed at the interface with the vacuum.

3.3.4. Low plasma $\beta$ shock tube test. This is another test from [9]. It consists of rarefactions into a region of low plasma $\beta$ (defined as $\beta=\frac{2 p}{\mathbf{B}^{2}}$ ). The initial data are given by $U=U_{l}$ for $x<0.5$, and $U=U_{r}$ for $x>0.5$, with $\gamma=5 / 3$ and

$$
\begin{aligned}
& \rho_{l}=1, \mathbf{u}_{l}=(-\hat{u}, 0,0), \mathbf{B}_{l}=(1,0.5,0), p_{l}=0.45 \\
& \rho_{r}=1, \mathbf{u}_{r}=(\hat{u}, 0,0), \mathbf{B}_{r}=(1,0.5,0), p_{r}=0.45 .
\end{aligned}
$$

We first fixed the the velocity at $\hat{u}=3.1$. There was little difference between the second order schemes here. MHWP and MHpP are observed to converge towards the reference solution in 

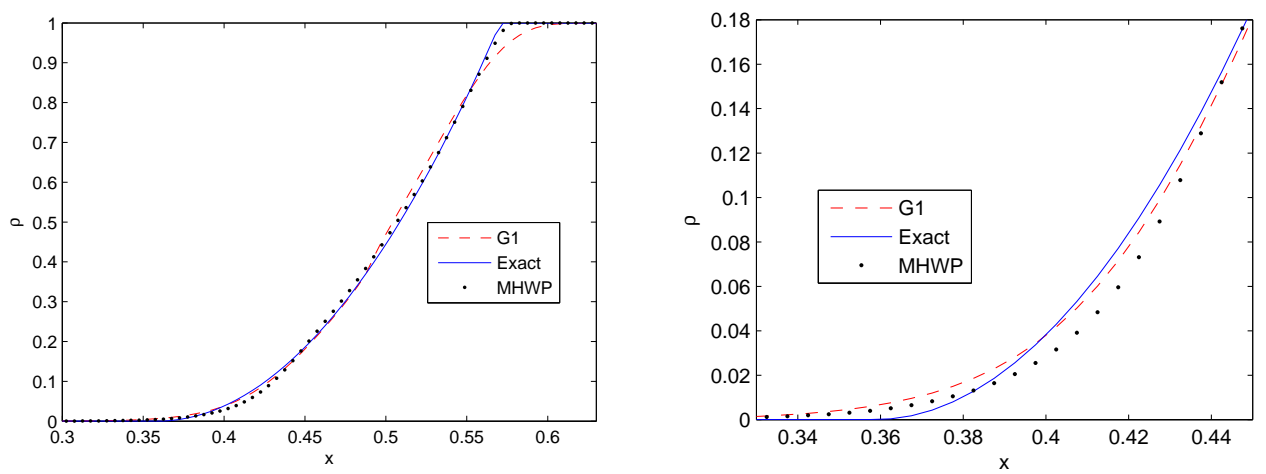

Figure 3.4. Vacuum test as computed by G1 and MHWP. The resolution was $h=0.005$, and $\rho$ is plotted at $t=0.1$. The right hand plot zooms in on the rarefaction tail.
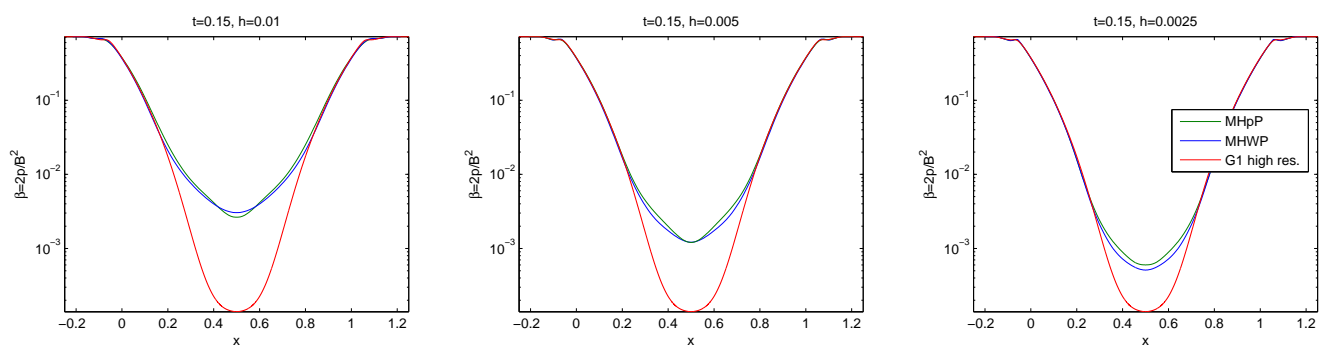

Figure 3.5. Refinement study of low $\beta$ test. The plots show plasma $\beta=\frac{2 p}{\mathrm{~B}^{2}}$ at time $t=0.15$, and resolutions are $h=0.01, h=0.005, h=0.0025$. The reference solution is computed by G1 at $h=2 \times 10^{-4}$.
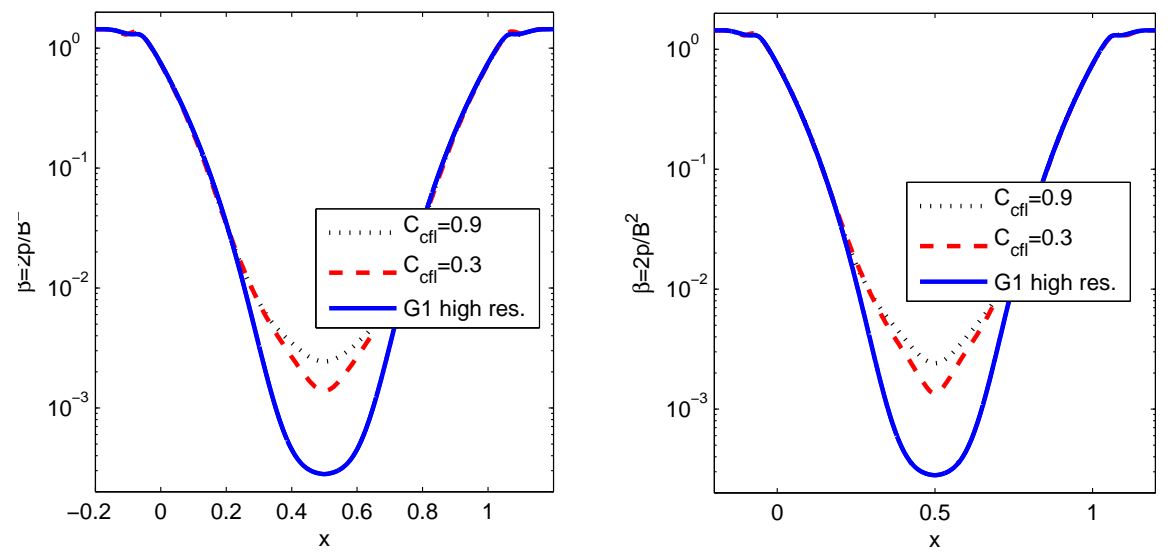

Figure 3.6. Comparison of CFL-numbers on low $\beta$ test. Plasma $\beta=\frac{2 p}{\mathbf{B}^{2}}$ is plotted. Left: MHWP, right: MHpP. The resolution was $h=0.005$.

Figure 3.5. The two schemes do equally well. The reference solution was computed using the first order scheme G1 at high resolution.

Although using a CFL-number of 0.9 worked well in all the test cases, it is interesting to see whether it makes a difference to follow the theoretical suggestion of using one third of the optimal value. From Figure 3.6 it seems that taking 0.3 as the CFL-number gives a slight improvement, 

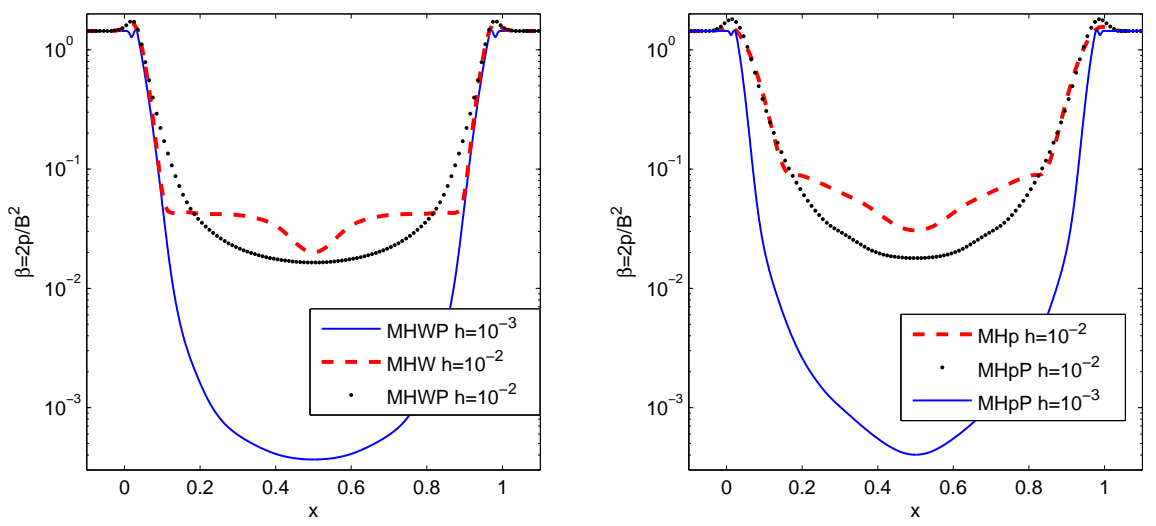

Figure 3.7. Comparison of positive and nonpositive schemes on low $\beta$ test. Plasma $\beta=\frac{2 p}{\mathrm{~B}^{2}}$ is plotted. Left: $\hat{u}=10.4$, right: $\hat{u}=14.2$.

but the difference is too marginal to justify the increased computational time.

Finally, we considered the limits of the stability ranges of the nonpositive schemes MHW and MHp with respect to the velocity $\hat{u}$. We computed until the time $t=\frac{1}{2}(\hat{u}+1.41)^{-1}$ (where 1.41 approximates the fast wave speed) at $h=0.01$. MHW failed for $\hat{u}>10.4$ while MHp ran until $\hat{u}>14.2$. The results are shown in Figure 3.7, where some differences in the low $\beta$ regions are visible. Somewhat surprisingly, the positive schemes reach the lowest $\beta$ values, but MHW does slightly better than MHWP in some other parts of the solution.

\section{Extension to MULTi-Dimensions}

To do multidimensional calculations, we need to be able to handle a varying $B_{n}$. A positive Riemann solver for this setting is given in [9], where the following modified equation for $\mathbf{B}$ is solved

$$
\mathbf{B}_{t}+\left(u \mathbf{B}-B_{n} \mathbf{u}\right)_{x}-\mathbf{u}\left(B_{n}\right)_{x}=0 .
$$

The resulting scheme is not conservative, hence the proof of Proposition 3.1 is not valid. We can write the scheme as

$$
\mathcal{S}_{\Delta t} U_{i}=U_{i}-\frac{\Delta t}{\Delta x}\left(\mathcal{F}_{l}\left(U_{i}, U_{i+1}\right)-\mathcal{F}_{r}\left(U_{i-1}, U_{i}\right)\right) .
$$

Incorporating this nonconservative numerical flux blindly into the MUSCL-Hancock scheme is not consistent with (4.1), as the source contribution is of vanishing size for smooth data. Also, this approach turns out to not be very stable. We will instead consider schemes of the form

$$
\mathcal{S}_{\Delta t} U_{i}=U_{i}-\frac{\Delta t}{\Delta x}\left(\mathcal{F}_{l}\left(U_{i}^{+}, U_{i+1}^{-}\right)-\mathcal{F}_{r}\left(U_{i-1}^{+}, U_{i}^{-}\right)\right)-\Delta t S_{i} .
$$

Extending nonconservative schemes to higher order in this manner is carried out in [1] and [7] for shallow water equations. For systems with a full energy equation, ensuring that positivity properties of first order schemes carry over requires more care. In order for the proof of 3.1 to hold, we need to take (dropping the index $i$ ) $S=S_{1}$ given by

$$
S_{1}=-\left(\mathcal{F}_{r}\left(U^{*}, U^{+}\right)+\mathcal{F}_{l}\left(U^{*}, U^{+}\right)-\mathcal{F}_{r}\left(U^{-}, U^{*}\right)+\mathcal{F}_{l}\left(U^{-}, U^{*}\right)\right) \frac{1}{h} .
$$

This gives a positive and consistent discretisation, but the source term is not necessarily second order accurate. The source $S_{1}$ may be computed as follows using the HLL3R solver. Note that we only get a source contribution for the magnetic field $\mathbf{B}$, so we write $S=\left(0,0,0,0,0, \mathbf{S}^{\mathbf{B}}\right)$.

(1) Find $U^{-}, U^{*}$ and $U^{+}$. 
(2) Calculate a signal velocity $\sigma$ for the interior problems by taking the maximum values allowed by the CFL condition:

$$
\sigma=C_{c f l} \frac{\Delta x}{\Delta t}
$$

(3) Calculate intermediate values of $\mathbf{u}$ at interior Riemann problems, denoted $\mathbf{u}_{S}^{ \pm}$, given by

$$
\begin{aligned}
& \mathbf{u}_{S}^{-}=\frac{\rho^{-}\left(u^{-}+\sigma\right) \mathbf{u}^{-}+\rho^{*}\left(\sigma-u^{*}\right) \mathbf{u}^{*}+\boldsymbol{\Pi}^{-}-\boldsymbol{\Pi}^{*}}{\rho^{-}\left(u^{-}+\sigma\right)+\rho^{*}\left(\sigma-u^{*}\right)} \\
& \mathbf{u}_{S}^{+}=\frac{\rho^{*}\left(u^{*}+\sigma\right) \mathbf{u}^{*}+\rho^{+}\left(\sigma-u^{+}\right) \mathbf{u}^{+}+\boldsymbol{\Pi}^{*}-\boldsymbol{\Pi}^{+}}{\rho^{*}\left(u^{*}+\sigma\right)+\rho^{+}\left(\sigma-u^{+}\right)},
\end{aligned}
$$

with

$$
\boldsymbol{\Pi}=\left(p+\frac{1}{2} \mathbf{B}^{2}-\left(B_{n}\right)^{2},-B_{n} B_{\perp}\right) .
$$

(4) Finally,

$$
\mathbf{S}_{1}^{\mathbf{B}}=\mathbf{u}_{S}^{-} \frac{B_{n}^{*}-B_{n}^{-}}{h}+\mathbf{u}_{S}^{+} \frac{B_{n}^{+}-B_{n}^{*}}{h} .
$$

The algorithm is more efficient than a full evaluation of the Riemann problems due to the simplicity of (4.5) and that we only need to know $\mathbf{u}_{S}^{ \pm}$. The consistency of $\mathbf{S}_{1}^{\mathbf{B}}$ with the source term $\mathbf{u}\left(B_{n}\right)_{x}$ may seen from setting $\mathbf{u}_{S}^{ \pm}=\mathbf{u}^{c}+O(h)$, yielding

$$
\mathbf{S}_{1}^{\mathbf{B}}=\mathbf{u}^{c} \frac{D B_{n}}{h}+O(h) .
$$

In most cases, we expect that the source term

$$
S_{2}=\left(0,0,0,0,0, \mathbf{u}^{c} \frac{D B_{n}}{h}\right) .
$$

may be used instead of $S_{1}$ to ensure efficiency and second order accuracy. The second order accuracy may be seen from rewriting the scheme so that the source is given by the more standard form

$$
\frac{1}{h} \mathbf{u}_{i}^{c}\left(B_{n, i}^{+}-B_{n, i-1}^{+}\right)_{u_{n}^{c}>0}+\frac{1}{h} \mathbf{u}_{i}^{c}\left(B_{n, i+1}^{-}-B_{n, i}^{-}\right)_{u_{n}^{c}<0}
$$

up to second order. The source discretisations $S_{1}$ and $S_{2}$ can be hybridised to also ensure positivity as follows.

(1) Pick some small energy value $\underline{\rho e}_{i}$, such as

$$
\underline{\rho e}_{i}=\mu \min \left((\rho e)_{i-1},(\rho e)_{i},(\rho e)_{i+1}\right), \quad \mu \in(0,1] .
$$

(2) Calculate the candidate $\tilde{U}$ using the source term $S_{2}$;

$$
\tilde{U}_{i}=U_{i}-\frac{\Delta t}{\Delta x}\left(\mathcal{F}_{l}\left(U_{i}^{+}, U_{i+1}^{-}\right)-\mathcal{F}_{r}\left(U_{i-1}^{+}, U_{i}^{-}\right)\right)-\Delta t S_{2, i} .
$$

(3) Calculate

$$
\xi_{i}=\left(1-\frac{\left((\tilde{\rho e})_{i}\right)_{+}}{\underline{\rho e}_{i}}\right)_{+}
$$

(4) Set

$$
\mathcal{S}_{\Delta t} U_{i}=\tilde{U}_{i}+\xi_{i} \Delta t\left(S_{1, i}-S_{2, i}\right) \text {. }
$$


We use $\mu=\frac{1}{2}$.

In the astrophysical fluid dynamics code FLASH $([15])^{1}$, the Powell term is included in the discrete form

$$
S_{F, i}=\left(0,0,0,0,0, \mathbf{u}_{i}^{c}\left[\left(B_{n}^{+}\right)_{i}+\left(B_{n}^{-}\right)_{i+1}-\left(B_{n}^{-}\right)_{i}-\left(B_{n}^{+}\right)_{i-1}\right] \frac{1}{2 h}\right),
$$

and the terms $S_{l}$ and $S_{r}$ are ignored (In fact, including them would violate second order accuracy). This scheme can also be made positive by using the hybridisation procedure described above.

Finally, for consistency $\Delta W$ should be modified to

$$
\begin{aligned}
\Delta \rho & =\frac{\Delta t}{2 h}(u D \rho+\rho D u) \\
\Delta u & =\frac{\Delta t}{2 h}\left(u D u+\frac{D p+B_{\perp} \cdot D B_{\perp}+B_{n} D B_{n}}{\rho}\right) \\
\Delta u_{\perp} & =\frac{\Delta t}{2 h}\left(u D u_{\perp}-\frac{B_{n} D B_{\perp}+B_{\perp} D B_{n}}{\rho}\right) \\
\Delta B_{n} & =\frac{\Delta t}{2 h} u D B_{n} \\
\Delta B_{\perp} & =\frac{\Delta t}{2 h}\left(u D B_{\perp}+D u B_{\perp}-B_{n} D u_{\perp}\right) \\
\Delta p & =\frac{\Delta t}{2 h}(u D p+\gamma p D u) .
\end{aligned}
$$

Other than that, the limiting inequalities of Proposition 3.2 and its corresponding algorithm remain unchanged.

4.1. Semidiscrete version. The semidiscrete case $(\Delta W=0)$ allows a simplification which is worth stepping aside to point out. The $\mathrm{W}$-reconstruction can be made positive in multi-dimensions if the following source term is added to $\mathbf{B}$

$$
\mathbf{S}=\left(\mathbf{u}+\frac{B_{n} D \mathbf{B}+D B_{n} \mathbf{B}}{\bar{c}_{l}+\bar{c}_{r}}\right) \frac{D B_{n}}{h}
$$

with the relaxation speeds

$$
\bar{c}_{l}=\rho^{-}\left(C_{c f l} \frac{\Delta x}{\Delta t}+u^{-}\right), \quad \bar{c}_{r}=\rho^{+}\left(C_{c f l} \frac{\Delta x}{\Delta t}-u^{+}\right)
$$

This result comes from modifying the proof of Proposition 3.1 by considering $U^{*+}$ and $U^{*-}$ such that

$$
4 U=U^{-}+U^{*-}+U^{*+}+U^{+}
$$

with $\mathbf{B}^{* \pm}=\mathbf{B}^{ \pm}, \rho^{* \pm}=\rho^{*},(\rho \mathbf{u})^{* \pm}=(\rho \mathbf{u})^{*}$ and

$$
E^{* \pm}=2\left(\frac{1}{2} \rho \mathbf{u}^{2}+\rho e\right)-\frac{1}{2}\left(\left(\frac{1}{2} \rho \mathbf{u}^{2}+\rho e\right)^{+}+\left(\frac{1}{2} \rho \mathbf{u}^{2}+\rho e\right)^{-}\right)+\frac{1}{2}\left(\mathbf{B}^{ \pm}\right)^{2}-\frac{1}{8} D \mathbf{B}^{2} .
$$

This modification of the argument reduces the number of Riemann problems with a source term contribution from 2 to 1 . The limiting inequalities that follow are

$$
\rho-\frac{1}{2}|D \rho|>0, p-\frac{1}{2}|D p|>0,
$$

and

$$
\frac{1}{8}\left(\rho+\frac{D \rho^{2}}{4 \rho}\right) D \mathbf{u}^{2}+\frac{1}{8} D \mathbf{B}^{2}<\frac{p}{\gamma-1} .
$$

\footnotetext{
${ }^{1}$ http://flash.uchicago.edu/website/home/
} 


\begin{tabular}{l|cc}
$h$ & MHWP2 & MHWP1 \\
\hline 0.02 & 0.127 & 0.126 \\
0.01 & 0.036 & 0.036 \\
0.05 & 0.0071 & 0.0074 \\
0.025 & 0.0012 & 0.0014
\end{tabular}

TABLe 5. One-dimensional test with variable $B_{n}$ : Errors in internal energy at $A=0.7$ given as $\Sigma_{i}\left|(\rho e)_{i}-(\rho e)\left(0, x_{i}\right)\right| h$.

4.2. Positivity in multi-dimensions. Our scheme may be extended to a uniform Cartesian multidimensional grid by applying it sequentially in each direction. This is known as the dimensional splitting method. In order to maintain second order accuracy, the order of the directions must be reversed between each time step (Strang splitting). Dimensional splitting obviously preserves the positivity properties of the scheme. An alternative method is to add the numerical flux contributions of each direction at once. Such a scheme may be written

$$
U^{n+1}=U^{n}-\frac{\Delta t}{h} \sum_{d} \Delta F^{d},
$$

where $d$ traverses the spatial dimensions, and $\Delta F^{d}$ are some flux differences. We rewrite this as

$$
U^{n+1}=\frac{1}{d} \sum_{d}\left(U^{n}-\frac{d \Delta t}{h} \Delta F^{d}\right) .
$$

Hence, if this scheme is positive in one dimension at CFL number $C_{1}$, it will be positive in $d$ dimensions at CFL-number $C_{1} / d$.

4.3. Numerical tests for multi-dimensional case. We now have several schemes to test in multidimensional settings. The time step is still chosen by (3.37). There are mainly two different source term discretisations to consider: i) The simple source $S_{2}$, yielding (4.12), denoted MHW2, and MHWP2 etc. ii) The schemes using (4.14), resulting from the hybrid of $S_{1}$ and $S_{2}$, are denoted MHWh and MHWPh etc. Setting $D W=0$ gives back the first order scheme G1 of [9].

4.3.1. One-dimensional smooth wave. First, we set up one-dimensional initial data with varying $B_{n}$. Although the data are unphysical, they provide a simple and adequate test for stability. A travelling wave solution to our Powell type system is given by $\mathbf{u}, B_{n} B_{\perp}$ and $p+\frac{1}{2} B_{\perp}^{2}-\frac{1}{2} B_{n}^{2}$ being constant. It has wave speed $u$. We choose $\rho=1, \mathbf{u}=(10,0,0), B_{n}=1-A \sin (2 \pi x)$. The pressure $p$ is set so that its minimum is 0.001 . The parameter $A$ may be chosen freely between zero and one. We try to compute one period (until $t=0.1$ ) and use periodic boundary conditions.

First fix $A=0.7$. MHWPh and MHWP2 did practically the same on this test. Table 5 shows errors at varying $h$. We also consider a scheme MHWP1 which uses $S=S_{1}$. Its performance is close to that of MHWP2, showing second order convergence. Internal energy profiles given by $\mathrm{MHWPh}$ are plotted in Figure 4.1. A valuable observation here was the necessity of the source term $S$ for positivity. Coupling $S=0$ with MHWP yielded a scheme that did not preserve positivity even at $A=0.01$. This suggests that the failure of Proposition 3.1 when $\left(B_{n}\right)_{x} \neq 0$ is a genuine feature. The schemes MHWP2 and MHpP2 were very stable in this test despite the lacking theoretical basis. We also tried out the source term (4.15). It had to be hybridised with MHWP1 to avoid negative pressures, and we denote the resulting scheme MHWPF. As shown in Figure 4.1, it produced strong spurious oscillations. One might suspect the oscillations were caused by the hybridisation, but they were present also in cases where MHWP1 was not invoked. 

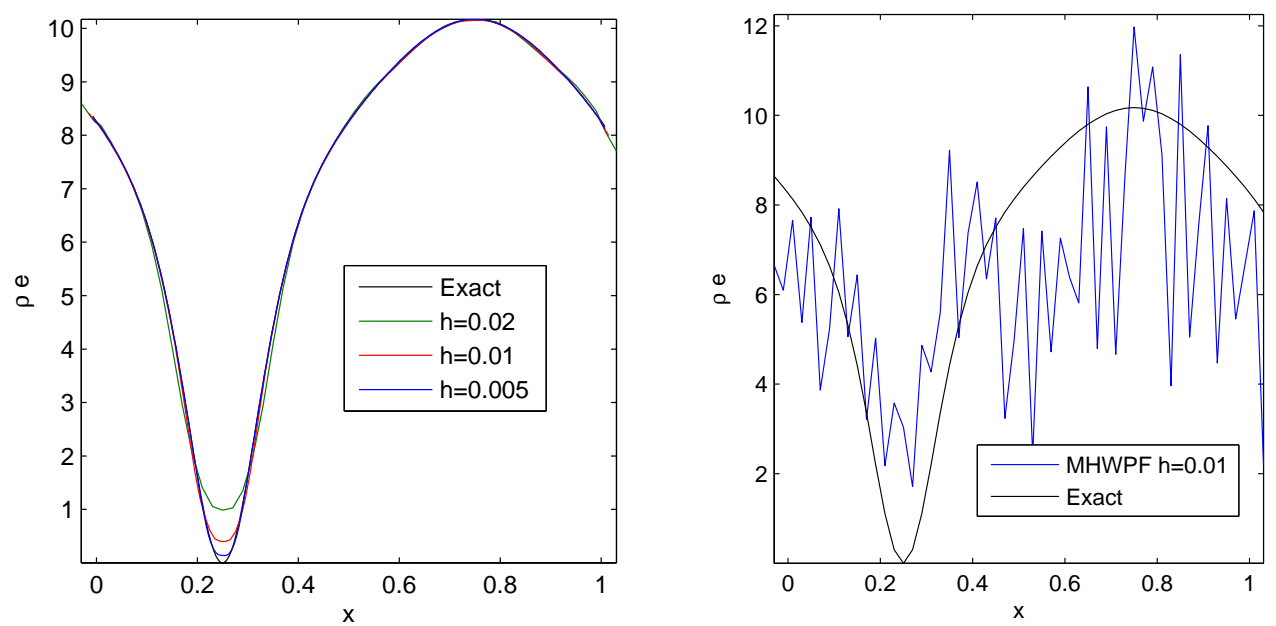

Figure 4.1. Left: One-dimensional test with variable $B_{n}$ computed by MHWPh at different resolutions. Right: The same test with MHWPF.

4.3.2. Rotor problem. This two-dimensional test problem, introduced in [2], involves low pressure values. The initial data are (denoting $\mathbf{u}=(u, v, w)$ and $\left.\mathbf{B}=\left(B_{x}, B_{y}, B_{z}\right)\right)$ as follows:

$$
\begin{gathered}
\rho=\left\{\begin{array}{ll}
10 & \text { if } r \leq 0.1, \\
1+9 \tilde{f}(r) & \text { if } r \in(0.1,0.115), \\
1.0 & \text { if } r \geq 0.115,
\end{array} \quad u= \begin{cases}-10 y+5 & \text { if } r \leq 0.1, \\
(-10 y+5) \tilde{f}(r) & \text { if } r \in(0.1,0.115), \\
0.0 & \text { if } r \geq 0.115,\end{cases} \right. \\
v= \begin{cases}10 x-5 & \text { if } r \leq 0.1, \\
(10 x-5) \tilde{f}(r) & \text { if } r \in(0.1,0.115), \quad w=0, \\
0.0 & \text { if } r \geq 0.115,\end{cases} \\
B_{x}=\frac{2.5}{\sqrt{4 \pi}}, \quad B_{y}=0, \quad B_{z}=0, \quad p=0.5 \\
r=\sqrt{(x-0.5)^{2}+(y-0.5)^{2}}, \quad \tilde{f}(r)=\frac{1}{3}(23-200 r) .
\end{gathered}
$$

We considered the computational domain given by $0 \leq t \leq 0.27$, and $(x, y) \in[0,1]^{2}$. We used directional splitting, and at the spatial boundary we implemented Neumann conditions with ghost cells. MHWh, MHWPh, MHWP2, MHpPh and MHpP2 were all found able to handle this case, and produced very similar results. We plotted the internal energy $\rho e$ at time $t=0.27$ in Figure 4.2 from $\mathrm{MHpPh}$ and $\mathrm{MHWPh}$.

To make the test more challenging we lowered the initial pressure to $p=10^{-8}$. In this case, MHWh could not get past the first time step. MHWPh, MHpPh, MHWP2 and MHpP2 were all stable also in this low $\beta$ case. They produced very similar results. In particular, the two types of central source term $S$ produced the same, hence we conclude that simply using $S_{2}$ gives a very stable scheme. We plotted the magnetic energy $\frac{1}{2} \mathbf{B}^{2}$ at $t=0.27$ from MHWP2 in Figure 4.3. This was complemented with a plot of magnetic field lines integrated from evenly spaced points along the $y$-axis. To compare the numerical dissipation of the schemes, we plotted the total internal energy $\int_{(0,1)^{2}} \rho e \mathrm{~d} x \mathrm{~d} y$ as a function of time, see Figure 4.4. In the first time step, the second order schemes were very similar to G1, a consequence of the virtually zero pressure. Already in the second time step, as the gas had been heated locally from dissipation, the second order schemes did better than G1. It seemed that MHpP2 was slightly less dissipative than MHWP2 in the early phases. An explanation for this could be that the limiting inequalities of Proposition 3.2 are less restrictive (at least as we implemented them). 

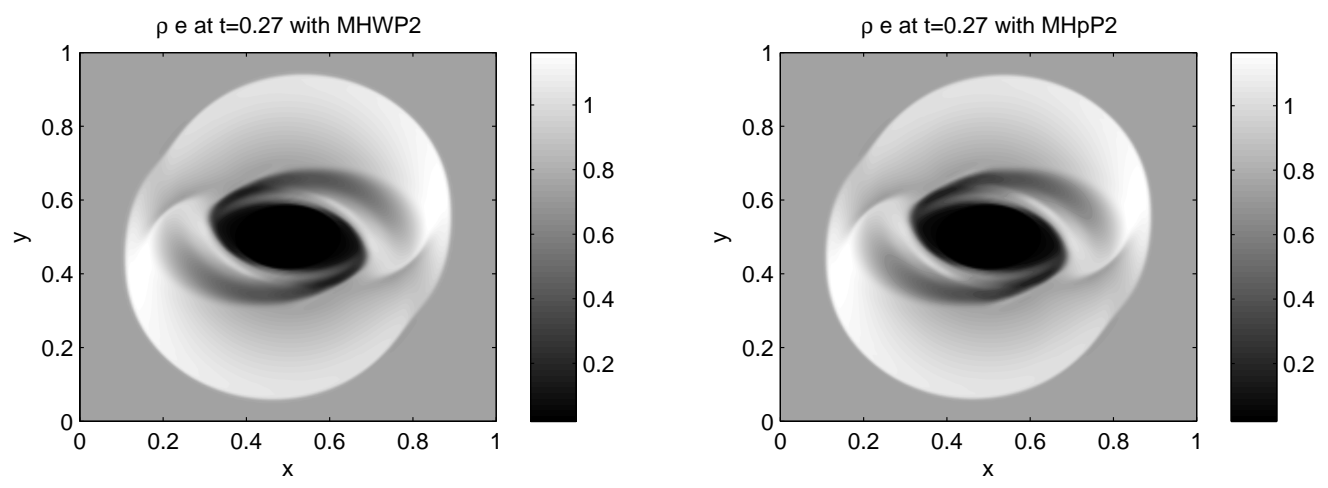

FiguRE 4.2. Contour plots of pressure from the rotor problem test results.
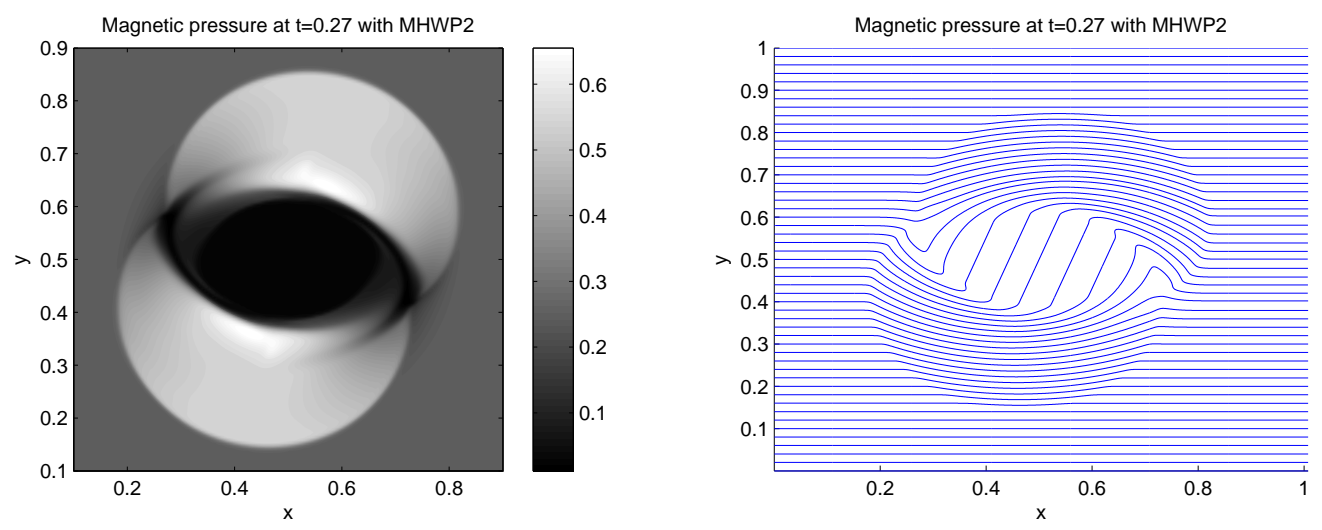

Figure 4.3. Results from low $\beta$ rotor problem at $t=0.27$. Left: Contour plot of magnetic pressure $\frac{1}{2} \mathbf{B}^{2}$ from MHWP2. Right: Magnetic field lines from MHWPh drawn with an intrinsic Matlab routine.

Finally, we compare the internal energy profiles produced with the different schemes in Figure 4.5. The second order schemes produced a sharper structure than G1. Again, hybridisation with a positive scheme was essential in MHWPF (using (4.15)). It reproduced the overall structure, but introduced small scale disturbances which we attribute to the oscillatory behaviour demonstrated in the previous test. This test demonstrated that we have increased the robustness range of the original scheme by several orders of magnitude. For example, MHWh failed already at an initial $p=10^{-2}$.

\section{Conclusion}

Presented in this paper is a method that, due to physically and mathematically relevant estimates, is significantly more robust than standard higher order methods. The basic ingredients of the scheme in one dimension are a positive and entropy stable approximate Riemann solver, and a new reconstruction procedure that preserves the good properties of the Riemann solver via a positivity proof. When generalising the positive scheme to more than one space dimension, it was crucial to make the one-dimensional method meaningful also when the restriction $\nabla \cdot \mathbf{B}=0$ was violated. We needed a novel upwind discretisation of Powell source terms to achieve this. We point out here that some schemes do not work well near vanishing pressure or density, because strict hyperbolicity is lost and characteristic decompositions become ill-posed. The approximate Riemann solver and reconstruction techniques used in this paper are robust with respect to this issue, which is an important reason for the overall stability of the scheme. 

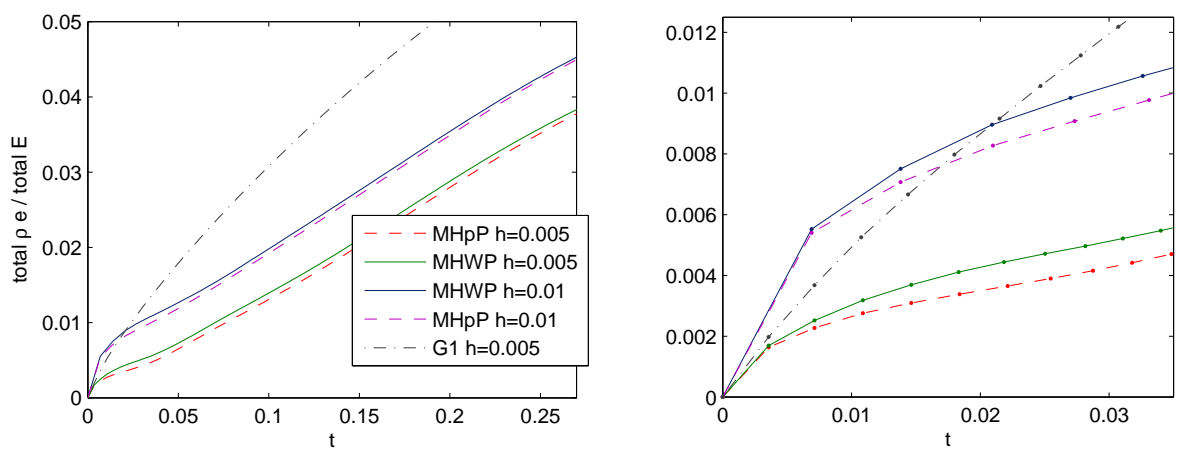

FiguRE 4.4. Low $\beta$ rotor problem: Time series of total internal energy relative to total energy. The right hand plot zooms in on the early phase.
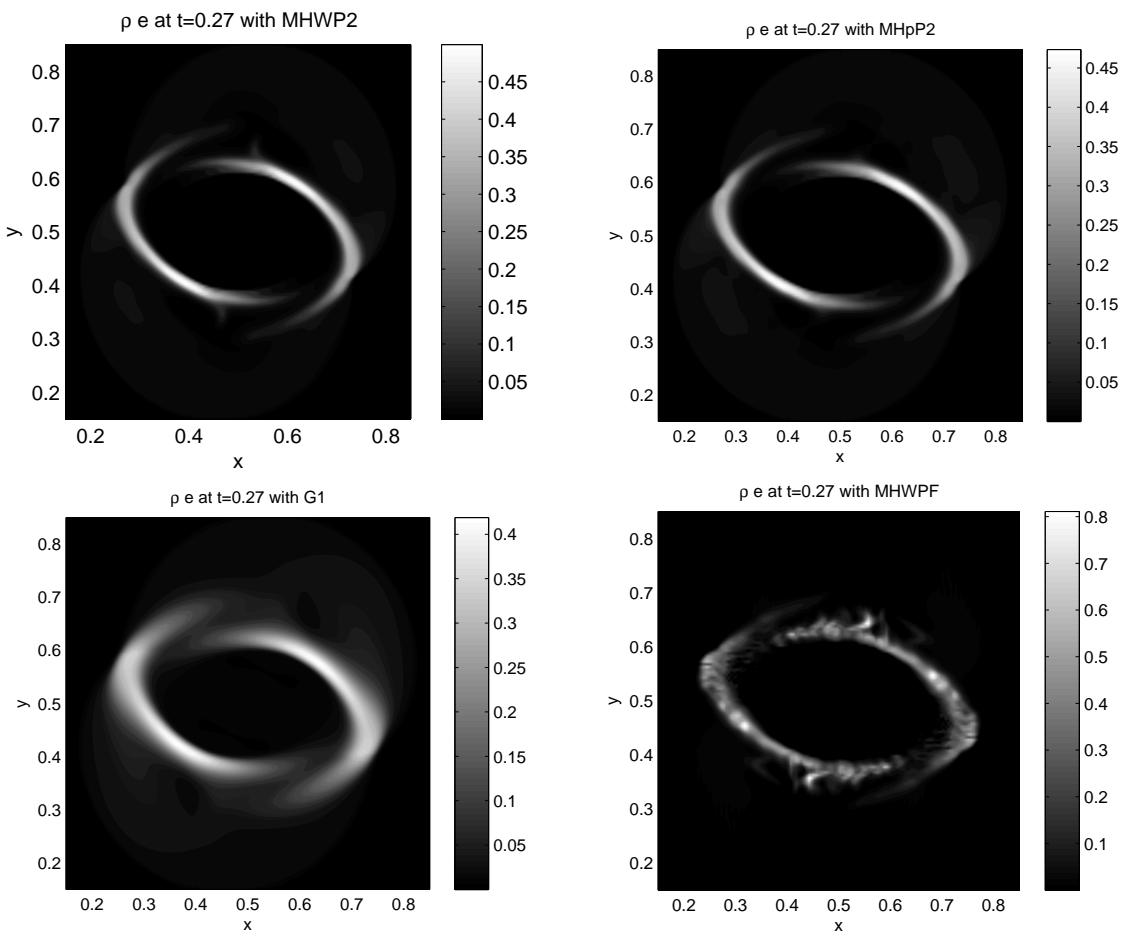

FiguRE 4.5. Low $\beta$ rotor problem: Pressure $p$ at $t=0.27$ and $h=0.005$ with different schemes. Left to right: $\mathrm{MHWPh}, \mathrm{MHpPh}, \mathrm{G} 1, \mathrm{MHWPF}$.

In order to ensure positivity of higher order schemes, it is sometimes necessary to limit the gradients more than in the standard TVD-type machinery. The gradient limitations approximately take the form $\rho D \mathbf{u}^{2}+D \mathbf{B}^{2}<O(\rho e)$. This inequality is satisfied as long as both the local relative Mach number, $|D \mathbf{u}|$ over sound speed, stays small enough, and the local relative plasma $\beta$, defined as $2 p / D \mathbf{B}^{2}$, stays large enough. These numbers depend on resolution, and for certain smooth flows refinement should be a remedy. However, the internal energy $\rho$ e can decrease significantly when resolution increases, due to reduced numerical dissipation. Our numerical examples demonstrated that including the extra limiting of the gradients gave very robust schemes. The accuracy of the original schemes was maintained in the cases where they worked. Among three different ways of determining the gradient $D W$, the new $p$-reconstruction and the primitive variable based $W$ reconstruction produced the best results. Among those two reconstructions there was no strong evidence in favor of one over the other, but the $p$-reconstruction led to somewhat simpler formulas. 
In less robust schemes it is sometimes necessary to a priori impose lower threshold values on the density and pressure. This violates conservation of mass and energy, and possibly consistency with the equations. In spite of these anomalies, lower thresholds can prevent codes from crashing, and give good results. We are not able to provide any general guidelines for using this technique, however. The threshold values need to be chosen heuristically for each application. We are under the impression that the use of lower thresholds and their values are not always reported in the literature. The scheme presented in this work conserves mass and energy, and does not rely on any application dependent small parameters.

For multidimensional MHD simulations, the inclusion of Powell type terms was crucial for the stability. The discretisation of the source terms was taken care of by the approximate Riemann solver HLL3R of [9], and a new second order discretisation method. Our approach to discretising the Powell terms should also be useful for handling more general source terms. The new discrete source combined with the new gradient limiting led to schemes that were stable in tests with very low plasma $\beta$ values and very high sonic Mach numbers. Summarising the tests of the source terms more specifically, the simple source discretisation (4.10) was found to be accurate and very stable. In contrast, a central discretisation of the source term (4.15) was found to produce spurious oscillations and negative pressures. Furthermore, we tested a more complicated source discretisation that we could prove ensured a positive scheme. It gave test results similar to those due to (4.10).

To sum up the paper, we have presented and tested a highly robust second order accurate numerical method for ideal MHD. In particular, the method can handle very low values of pressure and mass density. The scheme consists of the Riemann solver of [8]-[9] used in a MUSCL-Hancock framework, and two new numerical techniques: A reconstruction that ensures the positivity of density and pressure, and an upwind scheme for source terms. We expect that our scheme will be very useful in codes intended for a range of applications where low pressures or densities may be a problem.

Acknowledments. The National Center for Atmospheric Research is sponsored by the National Science Foundation. The author would like to thank Mark Miesch for helpful comments.

(Knut Waagan) High Altitude Observatory (HAO)

National Center of Atmospheric Research.

P.O. Box 3000, Boulder,

80307-3000 COLORADO, U.S.A

E-mail address: knutwa@ucar.edu 